Article

\title{
African financial markets in a storm: Cryptocurrency safe havens during the COVID-19 pandemic
}

\author{
Seyram Pearl Kumah1*, David Adjei Abbam², Ransford Armah³, and Appiah-Kubi Evelyn ${ }^{4}$ \\ 1 Wits Business School, Johannesburg, South Africa; spkumah@gmail.com \\ 2 Kumasi Metropolitan Assembly, Kumasi, Ghana; adjeidavid2002@yahoo.co.uk \\ 3 Institute of Chartered Accountants (Ghana); ransfordarmah@yahoo.co.uk \\ 4 Christ Apostolic University College, Ghana; adkubi@yahoo.com \\ * Correspondence: spkumah@gmail.com
}

Received: 17 February 2021; Accepted: 22 March 2021; Published: 6 April 2021

\begin{abstract}
The COVID-19 pandemic provides the first widespread bear market conditions since the inception of cryptocurrencies. We test the haven properties of cryptocurrencies for African stocks and commodity markets in a pandemic implementing the frequency domain spillover index. Data spans $11^{\text {th }}$ August 2015 to $28^{\text {th }}$ August 2020 at a daily frequency. Findings show weak interconnectedness across markets suggesting non-contagion risk and that cryptocurrency are safe havens for African stocks and commodity indices from the medium-term. We find the major transmitters of spillover effects across markets to be time-varying and heterogeneous. This study provides significant risk diversification benefits for policymakers and investors in the African financial markets.
\end{abstract}

Keywords: Cryptocurrency; African stock; Commodity; Safe havens; Spillover effects

JEL codes: C22; G11; G15; G32

\section{Introduction}

The finance literature provides evidence that the loss aversion of investors makes them extremely concerned with loss avoidance than related gains from investment and this motivates the concept of an investment haven (Tversky and Kahneman, 1991; Hwang and Satchell, 2010). In line with Baur and Lucey (2010), haven assets are negatively or uncorrelated with traditional assets in periods of market disruptions. The relationship between cryptocurrencies and the traditional financial markets works through the modern portfolio theory propounded by Markowitz (1952) which contends that adding an asset to a portfolio should be considered in the light of its impact on the performance and risk of the portfolio of assets. The empirical literature has established several haven assets including currencies (Sakemoto, 2018), gold (Baur and McDermott, 2010), and bonds (Flavin et al., 2014). In today's market, cryptocurrencies are digital assets with unique risk/reward profiles with a brand new level of correlation to traditional assets which can add a previously unattainable level of asset mix to an investor's holdings and impact the level of risk an investor has in a potentially positive way.

The exponential growth of cryptocurrencies has stirred several studies on their hedge, diversification, and have benefits for traditional assets. Notable studies in this regard include Bouri, Shahzad, and Roubaud (2019) who study the haven and hedging characteristics of eight virtual currencies against ten developed equity sectors. Kurka (2019) employs the cross-quantilogram technique to examine the nature of shock transmissions among stocks, commodities, financials, foreign exchange, and Bitcoin. Ünvana (2019) explores the effects of Bitcoin on Nikkei 225, BIST 100 index, S\&P 500, and SSE 380 stock markets using value-at-risk and causality analysis. Implementing the 
GARCH model, Al-Yahyaee, Mensi, Al-Jarrah, Hamdi, and Kang (2019) examine the ability of Bitcoin and gold as hedges for oil market volatility. Similarly, Adebola, Gil-Alama, and Madigu (2019) apply cointegration and fractional integration techniques to cryptocurrencies and gold prices and examined their short and long-term relationship. In another dimension, Ji, Bouri, Roubaud, and Kristoufek (2019) apply the transfer entropy technique to explore information interdependence among cryptocurrencies, agricultural, metals, and energy commodities. The evidence emerging from such studies highlights mixed and inconclusive findings for the asset markets.

While the pre-COVID findings are mixed, some of the studies evidenced the haven characteristics of cryptocurrencies for traditional assets. However, the hypothesis that cryptocurrencies have haven properties for traditional assets has not been extensively tested under extreme market situations due to the absence of a bear market in the historical pre-COVID samples. During the COVID-19 pandemic, studies including Conlon and McGee (2020) explore the haven benefits of Bitcoin for the S\&P 500 and find that Bitcoin increased the downside risk exposure for the international stock investor. In a similar dimension, Conlon, Corbert, and McGee (2020) investigate the haven characteristics of Bitcoin, Ethereum, and Tether for S\&P 500, FTSE 100, FTSE MIB, IBEX, and CSI 300 and finds that excerpt for Tether, Bitcoin and Ethereum are not safe havens for the stock markets studied. Ghorbel and Jeribi (2021) explore the association between the volatilities of five cryptocurrencies, gold, oil, VIX, Nasdaq, and S\&P500 implementing the BEKK-GARCH model and report a higher volatility spillover between cryptocurrencies and lower volatility spillover between cryptocurrencies and financial assets. The work of Jeribi and Manzli (2021) analyzes the behavior of cryptocurrencies concerning Tunisia's stock returns during the COVID-19 outbreak adopting the OLS regression. The findings suggest that Bitcoin act as a hedge and Ethereum was a diversifier for Tunisia's stock market before the COVID-19 outbreak.

In another study, Demir, Bilgin, Karabulut, and Doker (2020) scrutinize the connection between cryptocurrencies (Bitcoin, Ripple, Ethereum) and COVID-19 cases/deaths applying the wavelet coherence technique and finds that cryptocurrencies are hedges against COVID-19 death/cases. Iqbal, Fareed, Wan, and Shahzad (2020) examine the effect of the COVID-19 pandemic on the top ten cryptocurrency returns applying the Quantile-on-Quantile technique and finds that majority of the cryptocurrencies performed better against small shocks of COVID-19. Zaremba, Kizys, Tzouvanas, Aharon, and Demir (2021) study the behavior of 67 equity markets around the world during the COVID-19 outbreak implementing panel regression, machine learning techniques, and factor analysis. Findings indicate that stock markets in countries with low unemployment rates and populated with firms with conservative investment policies and low valuations relative to expected profits tend to be more resilient to the healthcare crisis. In another study, Zaremba, Kizys, Aharon, and Demir (2020) investigate the stringency of policy responses to the novel coronavirus pandemic in 67 countries around the world. The findings demonstrate that non-pharmaceutical interventions significantly increase equity market volatility and that information campaigns and public event cancellations are the major contributors to the growth of volatility.

There are, however, reasons to believe that haven properties may vary internationally as studies including Baker et al. (2016), Wang et al. (2019a), Wu et al. (2019), and Fang et al. (2019) document the independence of cryptocurrencies in monetary policy. Moreover, Bouri et al. (2018) also note that the state of an economy's financial market determines its relationship with cryptocurrencies. Therefore, we cannot assume that the relationship between cryptocurrencies and advanced markets applies to African markets. Thus, the motivation to model the returns of five large cryptocurrencies, four major African stock indices, one international stock index, and two global commodities and explore their time-varying connectedness. We uncover the haven benefits of the cryptocurrencies for downside risk management of the traditional assets implementing the frequency domain spillover index by Barunik and Krehlik (2018). This approach permits pairwise interconnectedness across the assets at different time scales which is more indicative of financial markets behavior. 
In general, we find a weak interconnectedness between crypto ${ }^{1}$-stocks, and crypto-commodity markets across frequencies suggesting non-contagion risk across the asset markets. This may provide significant risk diversification and hedging benefits for African stocks and commodity indices. Specifically, Ethereum and Tether are safe havens for the stocks from the medium-term, Ethereum is a haven for crude oil in the long-term, whiles Bitcoin and Litecoin are safe havens for gold in the longterm. The net transmitters and recipients of spillover effects across markets are time-varying and heterogeneous. Our finding is consistent with Conlon, Corbert, and McGee (2020), who finds that Tether has haven properties for the advanced stock market.

The remainder of the study is structured as follows. Section 2 covers a description of the methodology. Section 3 includes a description of data and statistical properties. Section 4 captures the results and discussions, and the last section is the conclusion and policy implications.

\section{Methodology}

We adopt the Barunik and Krehlik (2018) frequency domain spillover index technique for this study owing to its time-scale decomposition properties which are more indicative of associations in the financial market.

\subsection{Spillover frequency connectedness}

To explore the time-varying pairwise connectedness among cryptocurrencies, African stocks, and commodity indices, we implement Barunik and Krehlik's (2018) frequency domain spillover index which is based on Dew-Becker and Giglio (2016) spectral representations of variance decomposition. This methodology has a theoretical root to Diebold and Yilmaz (2012) and its time-frequency connectedness was first introduced by Barunik and Krehlik (2016) and extended by Barunik and Krehlik (2018).

As noted by Barunik and Krehlik (2016), the generalized impulse response function is decomposed considering the spectral behaviour of series $X_{t}$ as:

$$
S_{x}(w)=\sum_{h=0}^{\infty} E\left(X_{t} X_{t-h}\right) e^{-i h w}=\psi\left(e^{i h w}\right),
$$

where $w$ is frequency, $\infty$ is infinite horizon connectedness, and $\psi\left(e^{-i h w}\right)=\sum_{h=0}^{\infty} \psi\left(e^{-i h w}\right)$. The unconditional generalized forecast error variance decomposition (GFVED) is calculated on a particular frequency $w$ as:

$$
(\Theta(w))_{i, j}=\frac{\sigma_{j j}^{-1} \sum_{h=0}^{\infty}\left(\psi\left(e^{-i h w}\right) \sum\right)_{i, j}^{2}}{\sum_{h=1}^{\infty}\left(\psi\left(e^{-i h w}\right) \sum \psi\left(e^{i h w}\right)\right)_{i, j}}
$$

which is standardized to:

$$
(\Theta(w))_{i, j}=\frac{(\Theta(w))_{i, j}}{\sum_{j=1}^{k}(\Theta(w))_{i, j}} .
$$

We follow Barunik and Krehlik (2016) and express accumulative connectedness table over an arbitrary frequency band $d=(a ; b)$ as:

$$
\left(\Theta_{d}\right)_{i, j}=\int_{a}^{b}(\Theta(w))_{i, j} d w .
$$

We then specify the overall connectedness within a frequency band $d$ as:

$$
C^{d}=\frac{\sum_{i=1, i \neq j}^{k}\left(\theta_{d}\right)_{i, j}}{\sum_{i, j}\left(\theta_{d}\right)_{i, j}}=1-\frac{\sum_{i=1}^{k}\left(\theta_{d}\right)_{i, i}}{\sum_{i, j}\left(\theta_{d}\right)_{i, j}} .
$$

Within the spectral band $d=(a ;, b)$, strong connectedness is indicated when $C^{d}$ is close to unity. The contribution of a market $(i \neq j)$ to another $i$ on the spectral band $d$ is measured using the within from connectedness specified as:

\footnotetext{
${ }^{1}$ In this study crypto means cryptocurrencies.
} 


$$
C_{i \leftarrow}^{d}=\sum_{j=1, i \neq j}^{k}\left(\Theta_{d}\right)_{i, j} .
$$

whereas the contribution to a market $(i \neq j)$ from another $i$ on the spectral band $d$ is measured using the within to connectedness specified as:

$$
C_{i \rightarrow}^{d}=\sum_{j=1, i \neq j}^{k}\left(\Theta_{d}\right)_{i, j}
$$

Following Diebold and Yilmaz (2012), we measure total connectedness as $S g(H)=\sum_{d} C^{d}$ and pairwise connectedness as $\theta_{i j} \neq \theta_{j i}$.

\section{Data Description and Statistical Properties}

This paper explores the haven properties of cryptocurrencies for an international investor holding a position in a range of candidate African equity and commodity markets. Five cryptocurrencies ${ }^{2}$ (Bitcoin, Ethereum, Litecoin, Ripple, and Tether), five stock indices ${ }^{3}$ (EGX30, JSE, NSE, CSE for African markets, S\&P 500 for the international stock market), and two global commodities ${ }^{4}$ (Brent crude oil and Gold prices) are studied to achieve the set objectives. The cryptocurrencies, stocks, and commodities sampled are based on market capitalization and trading volume and can proxy for cryptocurrencies, African stocks, and commodity markets respectively. Data spans $11^{\text {th }}$ August 2015 to $28^{\text {th }}$ August 2020 at a daily frequency and are gleaned from Thomson Reuters Datastream and CoinMarketCap and expressed in a common currency (USD) to ease comparison and remove exchange rate noise. This practice has been justified in international financial market research (Pukthuanthong and Roll, 2009).

We calculate Monday-to-Friday returns for cryptocurrencies due to stocks and commodities not traded on weekends. We remove non-synchronous data points to prevent the problem of underestimation of true interconnectedness as did Martens and Poon (2001), and Das and Kannadhasan (2018). After matching the daily observations of the five cryptocurrencies with the five stock indices, and two commodities there were 1176 observations. The period of analysis and the number of observations in this study is constrained by the availability of cryptocurrency price data. All indices were then transformed into daily returns by taking the log difference.

The summary statistics of the asset returns are presented in Table 1 . The commodities show higher average returns and standard deviation relative to cryptocurrencies, and stocks suggesting the commodities as the most profitable and volatile assets of the panel. BTC, EGX30, NSE JSE, and S\&P 500 have negative skewness indicating dominant-negative returns than positive returns in the markets. Kurtosis statistics show that all the return series are leptokurtic, with significantly fatter tails and higher peaks indicating asymmetry. The Shapiro-Wilk test of normality for all the indices strongly rejects the null hypothesis that their distributions are normal. Further, the pictorial representation of the assets returns in Figure A1 in the Appendices exhibit fat tails, volatility clustering, and asymmetry suggesting nonlinearity. These features of the assets justify the use of asymmetric techniques which are more robust to the stylized facts to model their returns and examine their degree of interconnectedness across time.

\footnotetext{
2 Bitcoin, the best known, most widely traded cryptocurrency has the largest market capitalization. Ethereum is a decentralized computing platform that issues a reward for mining nodes. Ripple allows free business transactions by creating a currency and remittance network. Litecoin has an open-source aimed to preserve computing power and handle higher transaction volume. Tether is a stable coin, pegged to the US Dollar, meaning that it should maintain a 1-to-1 ratio with the U.S. dollar. This link is, however, not guaranteed by the issuer.

3 The African stock indices selected (EGX30-Egyptian stock exchange, JSE-Johannesburg stock exchange, NSE-Nigerian stock exchange, and CSE-Casablanca stock exchange) are the top four African stock markets and fairly represent the regions. We sample S\&P 500 to proxy for the international stock market.

4 The included commodities (Gold and Crude oil) are the primary exports of Africa even though the continent is dominated by several natural resources. They are produced on a commercial scale and have significance in international trade.
} 
Table 1. Summary Statistics of Cryptocurrencies, Stocks, And Commodity Returns

\begin{tabular}{ccccccccccccc}
\hline Statistics & BTC & ETH & LTC & XRP & USDT & EGX30 & CSE & NSE & JSE & S\&P 500 & CRUDE & GOLD \\
\hline Observ. & 1176 & 1176 & 1176 & 1176 & 1176 & 1176 & 1176 & 1176 & 1176 & 1176 & 1176 & 1176 \\
& & & & & & & & & & & & \\
Mean & 0.003 & 0.004 & 0.002 & 0.004 & 0.000 & 0.000 & 0.002 & 0.000 & 0.000 & 0.001 & 0.058 & 0.121 \\
Std. Dev. & 0.042 & 0.063 & 0.061 & 0.072 & 0.006 & 0.020 & 0.059 & 0.015 & 0.015 & 0.012 & 0.279 & 0.346 \\
Skewness & -0.222 & 0.748 & 2.237 & 2.780 & 0.890 & -4.892 & 33.450 & -3.152 & -0.185 & -0.728 & 0.742 & 1.819 \\
Kurtosis & 9.591 & 6.061 & 21.592 & 25.807 & 27.903 & 73.745 & 1134.623 & 48.066 & 1.888 & 20.105 & 23.125 & 3.058 \\
Normtest.W & 0.887 & 0.893 & 0.801 & 0.735 & 0.689 & 0.705 & 0.048 & 0.803 & 0.980 & 0.793 & 0.386 & 0.456 \\
Normtest.p & 0.000 & 0.000 & 0.000 & 0.000 & 0.000 & 0.000 & 0.000 & 0.000 & 0.000 & 0.000 & 0.000 & 0.000 \\
\hline
\end{tabular}

Note: BTC-Bitcoin, ETH-Ethereum, LTC-Litecoin, XRP-Ripple, USDT-Tether, EGX30-Egypt, NSE-Nigeria, JSESouth Africa, CSE-Morocco, S\&P500-USA.

\section{Results and Discussion}

Following Barunik and Krehlik (2018) approach as used by Tiwari, Cunado, Gupta, and Wohar (2018), and Qarni, Gulzar, Fatima, Khan, and Shafi (2019), we estimate a twelve variable VAR with two (2) lag lengths and construct the net pairwise spillover connectedness. Results are discussed in section 4.1.

\subsection{Pairwise spillover frequency connectedness}

The results for the pairwise directional spillover connectedness of the assets markets are displayed in Table 2. As noted by Barunik and Krehlik $(2016 ; 2018)$ the spillover table has 4 frequency bands, and we choose the first and second bands ${ }^{5}$ (freq. 1 and 2) as short-term connectedness, the third band ${ }^{6}$ (freq. 3) as medium-term connectedness, and the fourth band ${ }^{7}$ (freq. 4 ) as long-term connectedness of the markets. The net pairwise directional spillover effect from one market to another is measured by subtracting the second variable from the first (stock market-cryptocurrency market) and (commodity market - cryptocurrency market). A negative (positive) value depicts that the corresponding cryptocurrency is a net transmitter (receiver) of spillover effects.

Panels 1 to 5 of Table 2 shows the net pairwise directional connectedness of cryptocurrencies and stock returns, while panels 6 and 7 display the net pairwise directional connectedness between cryptocurrencies, gold, and crude oil returns. The markets are weakly connected across frequencies. Specifically, we observe a positive net connectedness between the Egyptian stock market and the cryptocurrencies in panel 1 of Table 2 except for EGX30-USDT at all frequencies, and EGX30-ETH at freq. 1 and 2. This indicates that cryptocurrencies are net receivers of spillover effects from EGX30 across frequencies except for Tether and Ethereum which are net spillover effects transmitters to EGX30. Ethereum is decoupled from EGX30 at freq.4 suggesting haven benefits for EGX30 in the longterm. From Panel 2, we observe that all cryptocurrencies are net recipients of spillover effects from the Casablanca stock market across frequencies except for Ethereum (CSE-ETH) which is a net transmitter of spillover effects to CSE at freq. 1. This finding depicts that cryptocurrencies cannot be safe havens for CSE as market discrepancies from CSE may transmit to the cryptocurrency market.

\footnotetext{
5 The spillover table for the first band (freq. 1) 3.14 to 0.79 roughly corresponds to 1 to 4 days, and the second band (freq. 2) 0.79 to 0.20 roughly corresponds to 4 to 16 days.

${ }^{6}$ The spillover table for the third band (freq. 3) 0.20 to 0.05 roughly corresponds to 16 to 64 days.

${ }^{7}$ The spillover table for the fourth band (freq. 4) 0.05 to 0.00 roughly corresponds to 64 days to infinity.
} 
Table 2. Net Pairwise Spillover of Cryptocurrencies, Stocks, and Commodity Returns

\begin{tabular}{|c|c|c|c|c|}
\hline Markets & Freq. 1 & Freq. 2 & Freq. 3 & Freq. 4 \\
\hline EGX30-XRP & 0.0087 & 0.0020 & 0.0005 & 0.0001 \\
\hline EGX30-USDT & -0.0032 & -0.0022 & -0.0007 & -0.0001 \\
\hline EGX30-LTC & -0.0004 & 0.0027 & 0.0017 & 0.0003 \\
\hline EGX30-BTC & 0.0013 & 0.0025 & 0.0010 & 0.0002 \\
\hline EGX30-ETH & -0.0130 & -0.0009 & 0.0001 & 0.0000 \\
\hline CSE-XRP & 0.0192 & 0.0081 & 0.0031 & 0.0005 \\
\hline CSE-USDT & 0.0129 & 0.0089 & 0.0032 & 0.0005 \\
\hline CSE-LTC & 0.0083 & 0.0047 & 0.0029 & 0.0005 \\
\hline CSE-BTC & 0.0070 & 0.0008 & 0.0003 & 0.0001 \\
\hline CSE-ETH & -0.0013 & 0.0035 & 0.0013 & 0.0002 \\
\hline NSE-XRP & -0.0015 & 0.0033 & 0.0020 & 0.0003 \\
\hline NSE-USDT & -0.0100 & 0.0003 & 0.0000 & 0.0000 \\
\hline NSE-LTC & 0.0138 & 0.0049 & 0.0034 & 0.0006 \\
\hline NSE-BTC & -0.0045 & -0.0108 & -0.0027 & -0.0004 \\
\hline NSE-ETH & -0.0203 & 0.0046 & 0.0029 & 0.0005 \\
\hline JSE-XRP & -0.0378 & 0.0015 & 0.0023 & 0.0004 \\
\hline JSE-USDT & -0.0047 & 0.0007 & 0.0000 & 0.0000 \\
\hline JSE-LTC & 0.0235 & 0.0079 & 0.0026 & 0.0004 \\
\hline JSE-BTC & 0.0353 & 0.0061 & 0.0012 & 0.0002 \\
\hline JSE-ETH & -0.0018 & -0.0011 & 0.0002 & 0.0000 \\
\hline S\&P500-XRP & 0.0270 & 0.0079 & 0.0041 & 0.0007 \\
\hline S\&P500-USDT & 0.0801 & 0.0000 & 0.0020 & 0.0004 \\
\hline S\&P500-LTC & 0.0895 & 0.0020 & 0.0023 & 0.0004 \\
\hline S\&P500-BTC & 0.1202 & 0.0152 & 0.0072 & 0.0012 \\
\hline S\&P500-ETH & -0.0042 & -0.0013 & -0.0001 & 0.0000 \\
\hline Crude-XRP & -0.0077 & -0.0034 & -0.0019 & -0.0003 \\
\hline Crude-USDT & -0.0080 & -0.0034 & -0.0012 & -0.0002 \\
\hline Crude-LTC & 0.0082 & -0.0076 & -0.0030 & -0.0005 \\
\hline Crude-BTC & 0.0002 & -0.0031 & -0.0016 & -0.0003 \\
\hline Crude-ETH & -0.0008 & 0.0001 & -0.0002 & 0.0000 \\
\hline Gold-XRP & 0.0125 & -0.0006 & -0.0006 & -0.0001 \\
\hline Gold-USDT & 0.0008 & -0.0034 & -0.0018 & -0.0003 \\
\hline Gold-LTC & -0.0096 & -0.0006 & -0.0001 & 0.0000 \\
\hline Gold-BTC & -0.0097 & -0.0010 & -0.0001 & 0.0000 \\
\hline Gold-ETH & 0.0180 & -0.0023 & -0.0008 & -0.0001 \\
\hline
\end{tabular}

Note: freq. 1 and 2 indicates short-term connectedness, freq. 3 and 4 indicate medium-, and long-term connectedness respectively. 
The Nigerian stock market and cryptocurrency markets connectedness at panel 3 shows positive net connectedness except for freq. 1 of NSE-XRP, NSE-USDT, NSE-ETH, and all frequencies of NSEBTC. This implies that except for the negative connectedness which shows the corresponding cryptocurrencies as net spillover effects transmitters to NSE, the NSE is the major net transmitter of spillover effects to cryptocurrencies across all frequencies and that cryptocurrencies cannot provide haven benefits for NSE except for Tether which shows no connection in the medium-, and long-terms. The Johannesburg stock market and cryptocurrency markets in panel 4 show positive net connectedness across frequencies except for freq. 1 of JSE-XRP, JSE-USDT, and freq. 1 and 2 of JSE-ETH suggesting that market disruptions from JSE can transmit to the cryptocurrency markets across time. We observe no connectedness between JSE-USDT (freq. 3 and 4), and JSE-ETH (freq. 4) suggesting that Tether and Ethereum are safe havens for the Johannesburg stock market in the medium-, and longterms. The net pairwise connectedness between S\&P 500 and the cryptocurrencies in panel 5 display positive net connectedness across frequencies except for S\&P500-ETH at freq. 1 to 3 . This shows the S\&P 500 as the major net transmitter of spillover effects and that price fluctuations in the S\&P 500 may affect the price of cryptocurrencies. Nevertheless, we find Ethereum (S\&P500-ETH) to be isolated from S\&P 500 at freq. 4 suggesting haven benefits for S\&P500 in the long-term.

With regards to the commodity market, it is evident that the pairwise connectedness between crude oil and cryptocurrencies in panel 6 depicts negative net connectedness across frequencies except for freq. 1 of Crude-LTC, Crude-BTC, and freq. 2 of Crude-ETH. This is indicative that the crude oil market is a net recipient of spillover effects from the cryptocurrency market and that cryptocurrencies cannot be safe havens for the crude oil market across time since price disruptions in the cryptocurrency market may spillover to crude oil market across time except for Ethereum which shows no association with crude oil in the long-term. Except for Litecoin and Bitcoin (Gold-LTC), and (Gold-BTC) at freq. 4 which is segmented from the gold market, negative net connectedness dominates gold and cryptocurrencies connectedness across frequencies as shown in panel 7. This is suggestive that except for Litecoin and Bitcoin which can provide haven benefits for gold market disruptions in the longterm, all other cryptocurrencies studied may transmit spillover effects to the gold market across time.

We can conclude from our result that the interconnectedness between pairs of cryptocurrencies and stock markets is mostly positive suggesting the stock markets as the major spillover effect transmitters to cryptocurrency markets which contradicts the findings of Ji, Bouri, Lau, and Roubaud (2019) and Trabelsi (2018) which shows cryptocurrencies including Bitcoin and Litecoin as the largest net transmitters of spillovers effects. However, the interconnectedness between pairs of cryptocurrencies and commodities is mostly negative indicating the cryptocurrencies as the major spillover effect transmitters to gold and crude oil markets providing evidence in support of the works of Ji, Bouri, Lau, and Roubaud (2019) and Trabelsi (2018). Nevertheless, the spillover effects across markets are weak across frequencies. This non-contagion nature of cryptocurrency markets provides significant risk diversification and hedging benefits for African financial markets. In particular, we find Ethereum and Tether as safe havens for African and advanced stock markets from the mediumterm which confirms the study of Conlon, Corbet, and McGee (2020) who reports Ethereum and Tither as having haven properties for international stock markets. Ethereum is a haven for the crude oil market in the long-term, whiles Bitcoin and Litecoin are safe havens for the gold market from the longterm.

\section{Conclusions and Policy Implication}

While cryptocurrencies are frequently part of the discussion regarding potential haven investments, empirical research on their relevance before the corona pandemic lacked a period of significant turmoil in global financial markets. This paper explored the time-varying interconnectedness between cryptocurrencies and traditional assets in the African financial markets capturing the COVID-19 bear market. We shed new light on the haven properties of cryptocurrencies for international and domestic investors in the African financial markets. Generally, our result indicates a weak interconnectedness between crypto-stocks and crypto-commodity markets across 
frequencies suggesting non-contagion risk which may provide significant risk diversification and hedging benefits for African financial markets and the advanced stock market. Specifically, Ethereum and Tether are safe havens for African and advanced stock market downturns from the medium-term, Ethereum is a haven for crude oil market turmoil in the long-term, whiles Bitcoin and Litecoin are safe havens for gold market disruptions from the long-term. Nonetheless, the net transmitters and receivers of volatility spillovers across markets are contingent on the frequency under consideration.

The empirical results of this study both support and contradict related literature. The results support the inter-market linkages between cryptocurrencies and the traditional financial markets evidenced by Bouri, Shahzad, and Roubaud (2019) and contradict Corbet, Meegan, Larkin, Lucey, and Yarovaya (2018) and Gil-Alana, Abakah, and Rojo (2020) who found no connection between the markets. The current study examined the time-varying interconnectedness between cryptocurrencies and African financial markets focusing on five large cryptocurrencies, four top African stock indices, one international stock index, and two global commodities. Future studies could examine the interconnectedness between other cryptocurrencies and financial markets not covered in this study to enhance our understanding of the inter-market linkages.

Author Contributions: Seyram Pearl Kumah conceptualized the research idea, gathered data, and wrote the manuscript. David Adjei Abbam, Ransford Armah, and Evelyn Appiah-Kubi provided writing assistance and helped with data estimation.

Funding: This manuscript did not receive any specific grant from funding agencies in the commercial, public, or not-for-profit sectors.

Conflicts of Interest: No potential conflict of interest was reported by the authors.

\section{Appendix A}

Figure A1. Time Series Plots for Cryptocurrencies, African Stocks, And Commodities Returns

Time series plots for selected cryptocurrencies
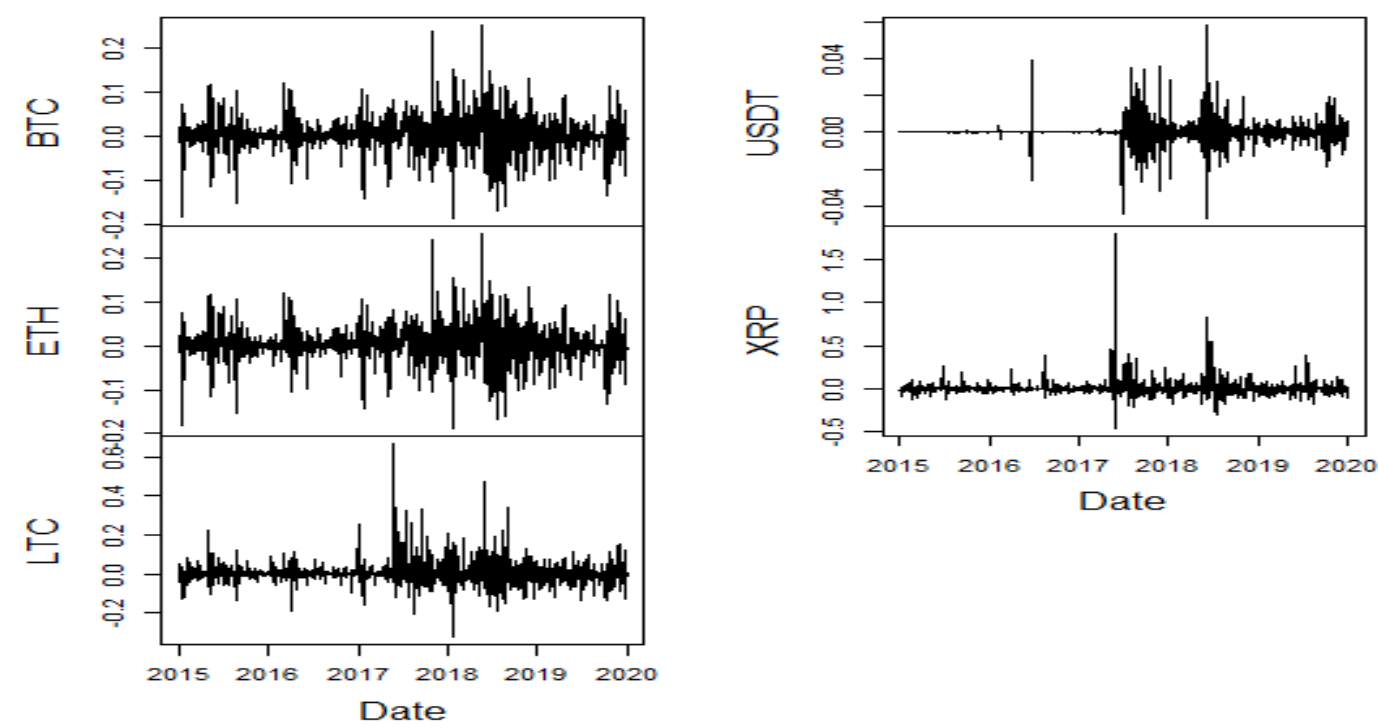
Time series plots for African stocks and commoditie:
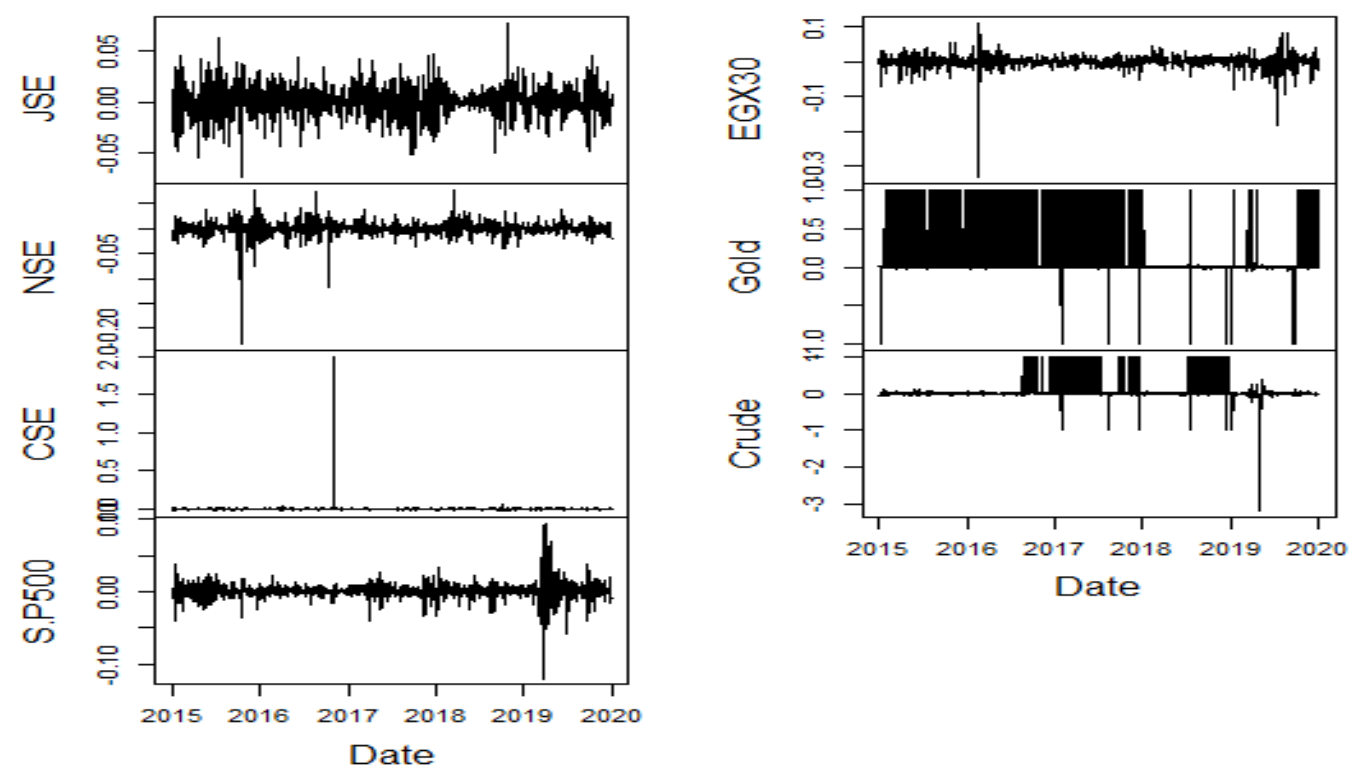

\section{References}

Adebola, S., S., Gil-Alana, A., L., \& Madigu, G. (2019). Gold Prices and the Cryptocurrencies: Evidence of Convergence and Cointegration. Physica A, 523, 1227-1236.

Al-Yahyaee, K., H., Mensi, W., Al-Jarrah, I., M., W., Hamdi, A., \& Kang, S., H. (2019). Volatility Forecasting, Downside Risk and Diversification Benefits of Bitcoin and Oil and International Commodity Markets: A Comparative Analysis with Yellow Metal. The North American Journal of Economics and Finance, 49, 104120.

Baker, S., R., Bloom, N., \& Davis, S., J. (2016). Measuring Economic Policy Uncertainty. The Quarterly Journal of Economics, 131(4), 1593-1636.

Barunik, J., \& Krehlik, T. (2016). Measuring the Frequency Dynamics of Financial and Macroeconomic Connectedness (No. 54). FinMaP-Working Paper.

Barunik, J., \& Křehlík, T. (2018). Measuring the Frequency Dynamics of Financial Connectedness and Systemic Risk. Journal of Financial Econometrics, 16(2), 271-296. https://doi.org/10.1093/jjfinec/nby001.

Baur, D., G., \& Lucey, B., M. (2010). Is gold a Hedge or a Safe Haven? An Analysis of Stocks, Bonds, and Gold. $v, 45(2), 217-229$.

Baur, D., \& Mc Dermontt, T., K., J. (2010). Is Gold a Safe Haven? International Evidence, Journal of Banking and Finance, 34(8), 1886-1898

Bouri, E., Shahzad, S., J., H., \& Roubaud, D. (2019). Cryptocurrencies as Hedges and Safe havens for US Equity Sectors. The Quarterly Review of Economics and Finance, 1-14. https://doi.org/10.1016/j.qref.2019.05.001.

Conlon, T., \& McGee, R. (2020). Safe Haven or Risky Hazard? Bitcoin during the COVID-19 Bear Market. Finance Research Letters, 101607.

Conlon, T., Corbet, S., \& McGee, R. J. (2020). Are Cryptocurrencies a Safe Haven for Equity Markets? An International Perspective from the COVID-19 Pandemic. Research in International Business and Finance, 101248.

Corbet, S., Meegan, A., Larkin, C., Lucey, B., \& Yarovaya, L. (2018). Exploring the Dynamic Relationships between Cryptocurrencies and Other Financial Assets. Economics Letters, 165, 28-34. doi.org/10.1016/j.econlet.2018.01.004.

Das, D., \& Kannadhasan, M. (2018). Do Global Factors Impact Bitcoin Prices? Evidence from Wavelet approach. Journal of Economic Research, 23, 227-264.

Demir, E., Bilgin, M. H., Karabulut, G., \& Doker, A. C. (2020). The Relationship between Cryptocurrencies and COVID-19 Pandemic. Eurasian Economic Review, 10(3), 349-360. 
Dew-Becker, I., \& Giglio, S. (2016). Asset Pricing in the Frequency Domain: Theory and Empirics. The Review of Financial Studies, 29(8), 2029-2068. https://doi.org/10.1093/rfs/hhw027.

Diebold, F. X., \& Yilmaz, K. (2012). Better to Give than to Receive: Predictive Directional Measurement of Volatility Spillovers. International Journal of Forecasting, 28(1), 57-66. https://doi.org/10.1016/j.ijforecast.2011.02.006.

Fang, L., Bouri, E., Gupta, R., \& Roubaud, D. (2019). Does Global Economic Uncertainty Matter for the Volatility and Hedging Effectiveness of Bitcoin? International Review of Financial Analysis, 61, 29-36.

Flavin, T., J., Morley, C., E., \& Panopoulou, E. (2014). Identifying Safe Haven Assets for Equity Investors through an Analysis of the Stability of Shock Transmission. Journal of International Financial Markets, Institutions, and Money, 33, 137-154.

Ghorbel, A., \& Jeribi, A. (2021). Investigating the Relationship between Volatilities of Cryptocurrencies and other Financial Assets. Decisions in Economics and Finance, 1-27.

Gil-Alana, L. A., Abakah, E. J. A., \& Rojo, M. F. R. (2020). Cryptocurrencies and Stock Market Indices. Are they related? Research in International Business and Finance, 51, 101063.

Hwang, S., \& Satchell, S., E. (2010). How Loss Averse are Investors in Financial Markets? Journal of Banking and Finance, 34 (10), 2425-2438.

Iqbal, N., Fareed, Z., Wan, G., \& Shahzad, F. (2020). Asymmetric Nexus between COVID-19 Outbreak in the World and Cryptocurrency Market. International Review of Financial Analysis, 73, 101613.

Jeribi, A., \& Manzli, Y. S. (2021). Can Cryptocurrencies be a Safe Haven during the Novel COVID-19 Pandemic? Evidence from the Tunisian Stock Market. Journal of Research in Emerging Markets, 3(1), 14-31.

Ji, Q., Bouri, E., Roubaud, D., \& Kristoufek, L. (2019). Information Interdependence among Energy, Cryptocurrency and Major Commodity Markets. Energy Economics, 81, 1042-1055.

Ji, Q., Bouri, E., Lau, C., K., M., \& Roubaud, D. (2019). Dynamic Connectedness and Integration in Cryptocurrency Markets. International Review of Financial Analysis.63, 257-272

Kurkaa, J. (2019). Do Cryptocurrencies and Traditional Asset Classes Influence each other? Finance Research Letters, $38,38-46$.

Markowitz, H. (1952). Portfolio Selection. The Journal of Finance, 7(1), 77-91.

Martens, M., \& Poon, S., H. (2001). Returns Synchronization and Daily Correlation Dynamics between International Stock Markets. Journal of Banking and Finance, 25(10), 1805-1827.

Pukthuanthong, K., \& Roll, R. (2009). "Global Market Integration: An Alternative Measure and Its Application." Journal of Financial Economics, 94 (2), 214- 232. DOI: 10.1016/j.jfineco.2008.12.004.

Qarni, M., O., Gulzar, S., Fatima, S., T., Khan, M., J., \& Shafi, K. (2019). Inter-markets Volatility Spillover in US Bitcoin and Financial Markets. Journal of Business Economics and Management, 20(3), 526-546.

Sakemoto, R. (2018). Do Precious and Industrial Metals act as Hedges and Safe Havens for Currency Portfolios? Finance Research Letters, 24, 256-262.

Tiwari, A., K., Cunado, J., Gupta, R., \& Wohar, M., E. (2018). Volatility Spillovers across Global Asset Classes: Evidence from Time and Frequency Domains. The Quarterly Review of Economics and Finance, 70, 194-202.

Trabelsi, N. (2018). Are There Any Volatility Spill-Over Effects among Cryptocurrencies and Widely Traded Asset Classes? Journal of Risk and Financial Management, 11(4), 66.

Tversky, A., \& Kahneman, D. (1991). Loss Aversion in Riskless Choice: A Reference-dependent Model. The Quarterly Journal of Economics, 106(4), 1039-1061.

Ünvana, Y. A. (2019). Impacts of Bitcoin on USA, Japan, China, and Turkey stock market indexes: Causality analysis with value at risk method (VAR). Communications in Statistics-Theory and Methods, 1-16.

Wang, G., J., Xie, C., Wen, D., \& Zhao, L. (2019). When Bitcoin meets Economic Policy Uncertainty (EPU): Measuring Risk Spillover Effect from EPU to Bitcoin. Finance Research Letters, 31.

Wu, S., Tong, M., Yang, Z., \& Derbali, A. (2019). Does Gold or Bitcoin Hedge Economic Policy Uncertainty? Finance Research Letters, 31, 171-178.

Zaremba, A., Kizys, R., Aharon, D. Y., \& Demir, E. (2020). Infected Markets: Novel Coronavirus, Government Interventions, and Stock Return Volatility around the Globe. Finance Research Letters, 35, 101597.

Zaremba, A., Kizys, R., Tzouvanas, P., Aharon, D. Y., \& Demir, E. (2021). The quest for multidimensional financial immunity to the COVID-19 Pandemic: Evidence from international stock markets. Journal of International Financial Markets, Institutions and Money, 71, 101284. 
(C) 2020 by the authors. This article is an open-access article distributed under the terms and conditions of the Creative Commons Attribution (CC BY) license (http://creativecommons.org/licenses/by/4.0/). 